\title{
THE MAGNETS OF THE METROLOGY LIGHT SOURCE IN BERLIN-ADLERSHOF*
}

\author{
P. Budz, M. Abo-Bakr, K. Bürkmann, V. Dürr, J. Kolbe, D. Krämer\#, J. Rahn and G. Wüstefeld, \\ BESSY GmbH, Berlin, Germany
}

\author{
I. Churkin, E. Semenov, S.Sinyatkin, A. Steshov, E. Rouvinskiy, \\ The Budker Institute of Nuclear Physics, SB RAS, Novosibirsk, Russia \\ Roman Markus Klein, Gerhard Ulm, \\ Physikalisch-Technische Bundesanstalt, Berlin, Germany
}

\section{Abstract}

The German National Metrology Institute (PTB) in close cooperation with BESSY II (Berlin) are currently carrying out the construction the low-energy "Metrology Light Source" (MLS) (Fig.1) as a synchrotron radiation facility situated in the close vicinity of BESSY II. Constructions of the MLS facility are in progress and nearly finished [1]. The user operation is scheduled to begin of 2008 .

Dedicated to metrology and technology development in the UV and EUV spectral range the MLS will close the gap that is existent since the shutdown of BESSY I [2].

A $100 \mathrm{MeV}$ microtron delivered by Danfysik A/S in Jyllige (Denmark) will provide the electrons for the MLS. The total circumference of the MLS is $48 \mathrm{~m}$. The MLS has two long and two short straight sections. It is a asymmetric double bend achromat. The electron energy is ramped to the desired value between $200 \mathrm{MeV}$ and 600 $\mathrm{MeV}$. The expected life time for electron energy between $200 \mathrm{MeV}$ and $600 \mathrm{MeV}$ at maximum beam current of 200 $\mathrm{mA}$ is between 1 hour and 10 hour, respectively.

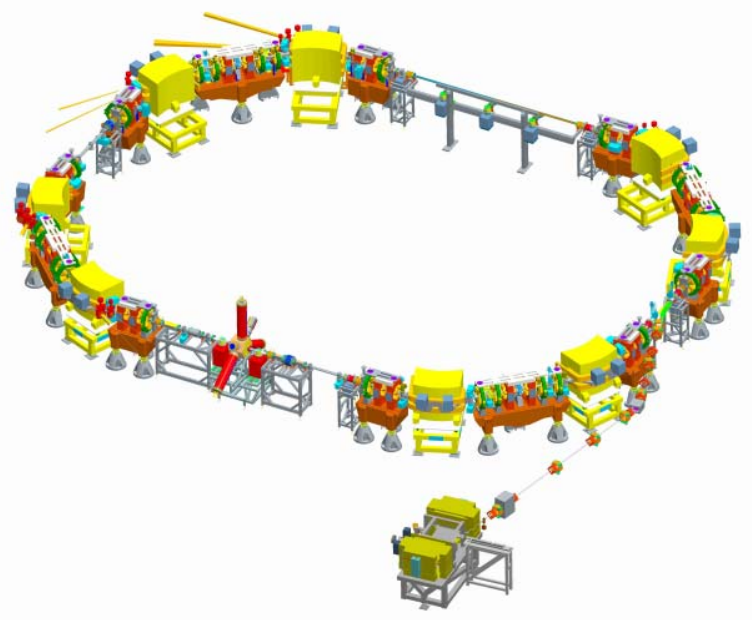

Figure 1: View of the MLS ring.

\footnotetext{
* Work supported by PTB, Abbestr. 2-12, 10587 Berlin (Germany)

\# now at GSI, Darmstadt (Germany)
}

The MLS magnetic ring (Fig. 1) consists of 8 bending magnets (yellow), 24 quadrupole magnets (red), 24 sextupole magnets (green) and 4 octupole magnets (black). The contract for the MLS magnets has been awarded to the Budker Institute for Nuclear Physics, Novosibirsk (Russia) [3].

A description of the MLS magnets based on the results of the factory acceptance tests (FAT) should be presented. The FAT is based on specified requirements: extensive coil tests, mechanical measurements of relevant dimensions. Magnetic measurements are performed for one magnet of each type. Tight tolerated dimensions should leads to similar magnetically properties.

\section{INTRODUCTION STORAGE RING MAGNET DESIGNS}

\section{Dipole Magnets}

For the MLS storage ring laminated, $\mathrm{C}$ - shaped curved dipole magnets with parallel pole shoes are used in the isomagnetic lattice structure. Fig. 2 gives a view of the bending magnet. The magnet of length $1_{\text {mech }}=1108 \mathrm{~mm}$ bend the beam on a radius of curvature of $1528 \mathrm{~mm}$. The specifications are listed in Tab.1. Each magnet is equipped with a pair of trim coils wound onto two of the "pancake"- coils (both orientated to the gap inner side) to allow horizontal beam steering. Operating field is up to $1.5 \mathrm{~T}$. The yoke is made by a composite technology from insulated laminations of $0.5 \mathrm{~mm}$ thickness. The welded core is compressed by two glued end packets.

Steel of type M940-50 from EBG Bochum (Germany) is used for the laminations. A chamfer of $45^{\circ} \times 13 \mathrm{~mm}$ is applied. The main content of higher harmonics in the integrated field comes from the sextupole components with an value $\mathrm{m}^{*} \mathrm{~L}<-0.8 \mathrm{~m}^{-2}$. Rectangular OFHC copper conductor of $21 \times 12 \mathrm{~mm}^{2}$ in size from Outokumpu (Finland) is used for the winding of the six "pancakes" with 14 windings each. 


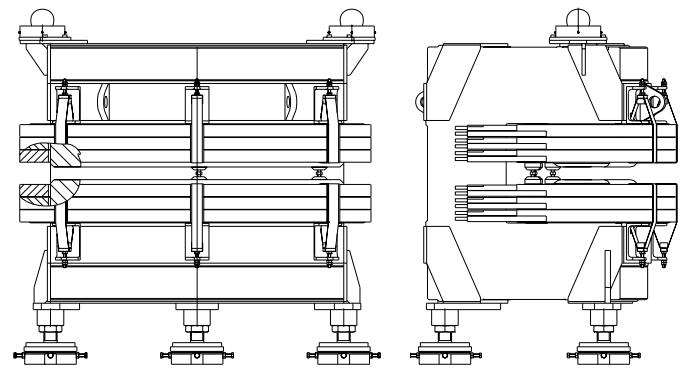

Figure 2: Front and side view of the dipole magnet.

\begin{tabular}{|l|l|}
\hline \multicolumn{2}{|c|}{ SR Dipole Magnet } \\
\hline \multicolumn{2}{|c|}{ Curved C shaped type } \\
\hline Gap & $50 \mathrm{~mm}$ \\
Field range & up to $1.5 \mathrm{~T}$ \\
Good field area & $60 \times 36 \mathrm{~mm}^{2}$ \\
Homogeneity dB/B & $2.5 \times 10^{-4} \mathrm{~T}$ \\
Current I & $630 \mathrm{~A}$ \\
Resistance R & $21 \mathrm{~m} \Omega$ \\
Inductance L & $38 \mathrm{mH}$ \\
Power Consumption P & $8 \mathrm{~kW}$ \\
\hline
\end{tabular}

Table 1: Design parameters for the storage ring dipole magnets.

At magnetic fields of $1.3 \mathrm{~T}$ corresponding to nominal full beam energy of $600 \mathrm{MeV}$, the saturation is $2 \%$.

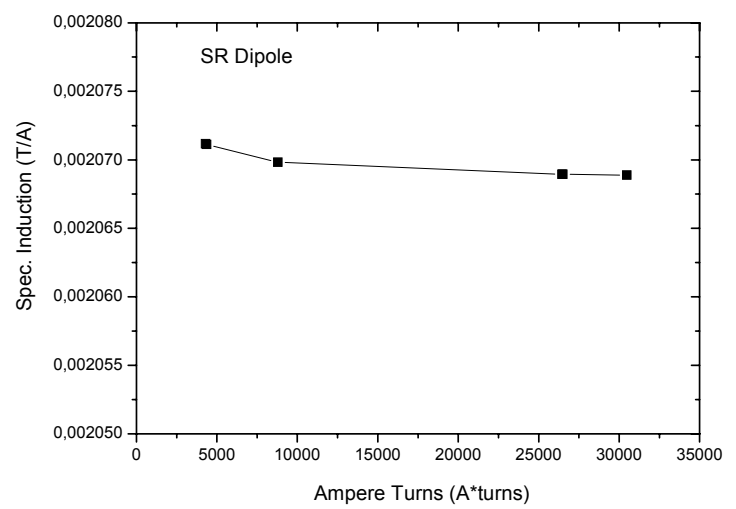

Figure 3: Measured excitation curve of the dipole field.

\section{Quadrupole Magnets}

There are 24 identical quadrupole lenses integrated in the isomagnetic lattice structure. Steel of type M940-50 with thickness of $0.5 \mathrm{~mm}$ from EBG Bochum (Germany) is used for the yokes of $165 \mathrm{~mm}$ length. According to the good performances of the BESSYII SR quadrupoles the laminations are shaped in the same way. Therefore the magnets are "figure of eight"-type with mirror symmetrical spacers from soft magnetic steel to shield the magnet centre from external fields [5]. A chamfer of $45^{\circ} \times 5.3 \mathrm{~mm}$ is applied to cancel the dominant systematic harmonics within the good field radius.

A rectangular hollow OFHC copper conductor of $6.5 \times 6.5 \mathrm{~mm}^{2}$ in size from Outokumpu (Finland) is used for the main coils, which are equipped with trim coils respectively. Figure 4 gives a view on the storage ring quadrupole. The specifications are listed in Tab.2.
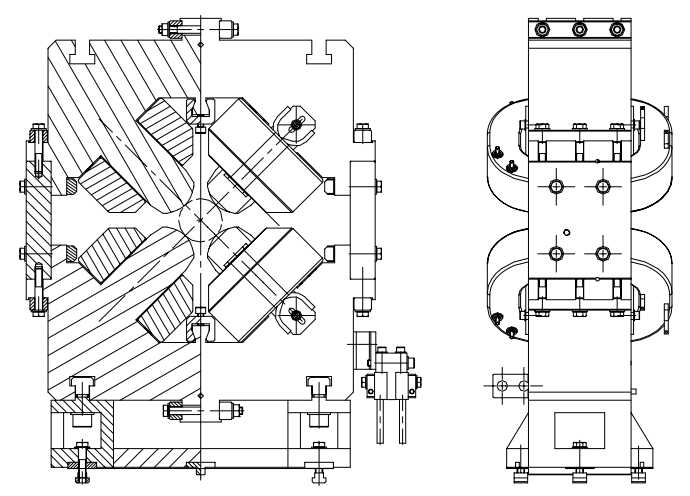

Figure 4: Front and side view of the quadrupole magnet.

\begin{tabular}{|l|l|}
\hline \multicolumn{2}{|c|}{ SR Quadrupole Magnet } \\
\hline \multicolumn{2}{|c|}{ "Figure of eight" -type } \\
\hline Aperture radius R & $35 \mathrm{~mm}$ \\
Core length L & $165 \mathrm{~mm}$ \\
Gradient G & $13 \mathrm{~T} / \mathrm{m}$ \\
Good field radius r & $30 \mathrm{~mm}$ \\
Homogeneity dGL/GL & $\leq 2 * 10^{-3}$ \\
Current I & $93 \mathrm{~A}$ \\
Resistance R & $122 \mathrm{~m} \Omega$ \\
Inductance L & $36 \mathrm{mH}$ \\
Power Consumption P & $1 \mathrm{~kW}$ \\
\hline
\end{tabular}

Table 2: Design parameters for the storage ring quadrupole magnets.

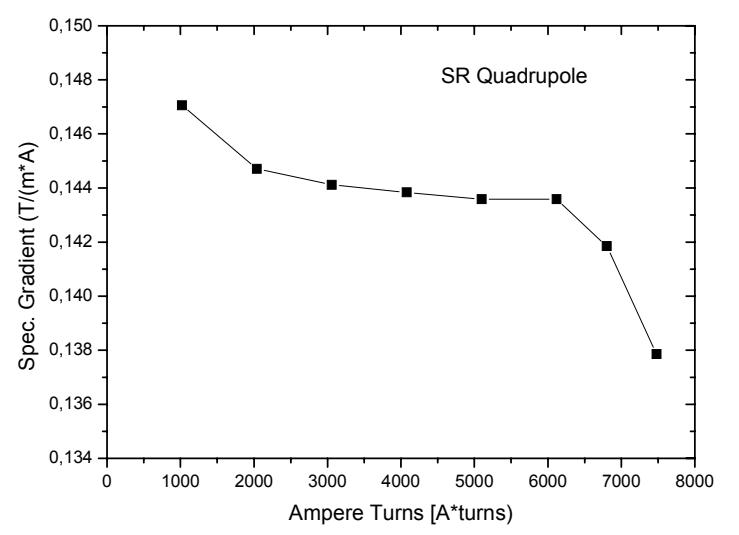

Figure 5: Measured excitation curve of the quadrupole field. 


\section{Sextupole Magnets}
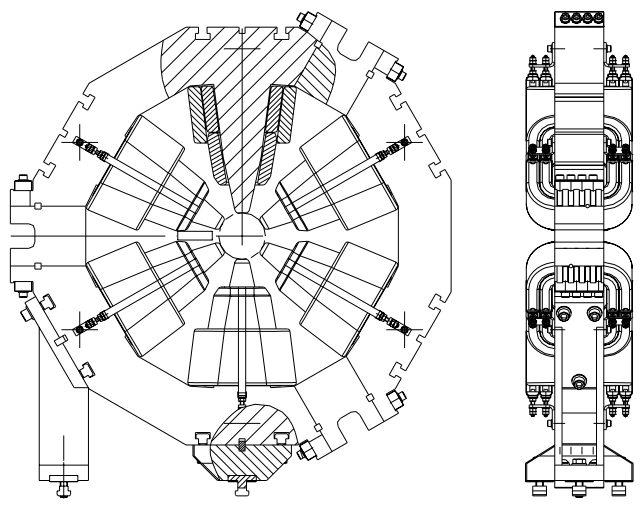

Figure 6: Front and side view of the sextupole magnet.

There are 24 identical sextupoles used as chromatic and harmonic correctors in the isomagnetic lattice structure. Together with a family of 4 octupole magnets (see also section D.), the sextupole magnets, grouped into 3 families, ensure the control of the nonlinear terms of the "momentum compaction"-factor. Figure 6 gives a view on the storage ring sextupole.

Steel of type M940-50 with thickness of $0.5 \mathrm{~mm}$ from EBG is used for stacking identical yokes of $80 \mathrm{~mm}$ length. Massive soft magnetic spacers are used to minimize irregular harmonics during dipolar correction. A chamfer of $45^{\circ} \times 6 \mathrm{~mm}$ is applied.

A $4.3 \times 3.5 \mathrm{~mm}^{2}$ rectangular OFHC copper conductor is used for the main coils. Two additional coils are integrated on each pole shoe to excite the sextupole magnets as horizontal and alternatively as vertical dipole correctors, respectively as skew quadrupole. Tab. 3 summarize the main parameters.

\begin{tabular}{|l|l|}
\hline \multicolumn{2}{|c|}{ SR Sextupole Magnet } \\
\hline Aperture radius R & $38 \mathrm{~mm}$ \\
Core length L & $80 \mathrm{~mm}$ \\
Gradient G & $280 \mathrm{~T} / \mathrm{m}$ \\
Good field radius $\mathrm{r}$ & $30 \mathrm{~mm}$ \\
Homogeneity dGL/GL & $\leq 2^{*} 10^{-3}$ \\
Current I & $93 \mathrm{~A}$ \\
Resistance R & $204 \mathrm{~m} \Omega$ \\
Inductance & $36 \mathrm{mH}$ \\
Power consumption P & $240 \mathrm{~W}$ \\
\hline
\end{tabular}

Table 3: Design parameters for storage ring sextupole magnets.

\section{Octupole Magnets}

On the basis of the presented materials, one family of 4 identical octupole magnets are used for the ring. Fig. 8 gives a view on the storage ring octupole.

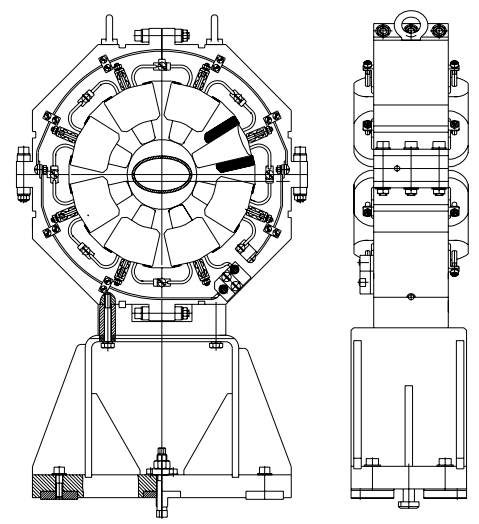

Figure 8: View of the octupole magnet.

\section{OUTLOOK}

All multipole magnets will be installed with high precision slots on the surfaces of the cast iron girders. The adjustment of the magnets will be done with two Taylor Hobson Balls mounted on the overall cross bar. Since all multipole magnets and girders are delivered, the assembly already started. Dipoles will be installed on movable support construction for an easy handling of the vacuum chamber. FAT and delivery of the dipole magnets is in preparation.

\section{REFERENCES}

[1] K Bürkmann et al., "Status of the Metrology Light Source", Proc. EPAC06.

[2] R. Klein et al., "The Metrology Light Source- An Electron Storage Ring Dedicated To Metrology“, Proc. EPAC06.

[3] Technical specification for production and supply of the MLS Magnets, BESSY, Berlin,2004.

[4] Inspection reports for the MLS Magnets, BINP, Novosibirsk 2006.

[5] Th. Becker at al., "Prototype Development of the BESSY II SR Magnetic Elements", Proc. EPAC 96. 